\title{
Computation of Multi-Dimensional Material Response Coupled to Hypersonic Flow
}

\author{
Jonathan E. Wiebenga* and Iain D. Boyd ${ }^{\dagger}$ \\ University of Michigan, Ann Arbor, Michigan, 48109
}

\begin{abstract}
Development of a material response model for non-charring ablative materials and twodimensional/axisymmetric geometries is presented. This model is loosely coupled to a computational fluid dynamics code as a boundary condition subroutine to allow for prediction of the fluid/surface interactions that occur for an ablating material in hypersonic flow. This coupled solution technique is applied to the case of the IRV-2 vehicle and axisymmetric material response results are compared with a similar coupling procedure that uses a one-dimensional material response code. The effect of multidimensional material response appears to have little impact on the flow field for this case, but the in-depth temperature profiles of the IRV-2 vehicle are considerably different when comparing the axisymmetric and one-dimensional results.
\end{abstract}

\section{Nomenclature}

$B_{c}^{\prime} \quad$ Dimensionless mass blowing rate

$C_{h} \quad$ Aerodynamic heat transfer coefficient

$C_{m} \quad$ Mass transfer coefficient

$N \quad$ Number of nodes in mesh

$N_{e} \quad$ Number of elements in mesh

$R \quad$ Radius

$T \quad$ Temperature

$\Delta t \quad$ Time step

$\dot{m} \quad$ Mass flux

$\dot{s} \quad$ Surface recession rate

$\epsilon \quad$ Emissivity

$\overline{\mathbf{k}} \quad$ Thermal conductivity tensor

$\dot{\mathbf{q}}^{\prime \prime} \quad$ Internal heat flux vector

$\hat{\mathbf{n}} \quad$ Unit outward normal vector

v Velocity

$\nu \quad$ Poisson's ratio

$\rho \quad$ Density

$\sigma \quad$ Stefan-Boltzmann constant

e Energy

$\begin{array}{ll}h & \text { Enthalpy } \\ i & \text { Trajectory point index } \\ n_{x}, n_{y} & \text { Components of normal vector } \\ p & \text { Pressure } \\ q_{C F D} & \text { Heat flux from CFD } \\ t & \text { Time } \\ u, v & \text { Nodal displacement } \\ x, y & \text { Coordinates } \\ \text { Subscripts } \\ \infty & \text { Freestream quantity } \\ a h & \text { Aerodynamic heating } \\ c a l c & \text { Calculated value } \\ c s & \text { Control surface } \\ \text { exact } & \text { Exact value } \\ o & \text { Initial or reference condition } \\ r & \text { Recovery quantity } \\ r o t & \text { Rotational } \\ s & \text { Solid quantity } \\ v e & \text { Vibrational-electronic } \\ w & \text { Wall quantity }\end{array}$

\section{Introduction}

$\mathrm{H}$ YPERSONIC vehicles are subjected to high heat loads throughout their flight trajectories, and as a result, some form of thermal protection system (TPS) is required to ensure vehicle survival. Since it can be very costly and difficult to experimentally replicate the flow conditions to which these vehicles are subjected, accurate prediction of the behavior of TPS materials is crucial to the efficient design of a hypersonic flight vehicle.

\footnotetext{
*Graduate Student, Department of Aerospace Engineering, AIAA Student Member.

$\dagger$ Professor, Department of Aerospace Engineering, Fellow AIAA
} 
Ablative materials can generally be divided into charring and non-charring, or surface, ablators. Surface ablators, as the name suggests, only lose mass at their surface and, unlike charring ablators, do not undergo in-depth decomposition or pyrolysis. As the ablator heats up, boundary layer gases begin to react with the surface causing the chemical removal of material. This in turn leads to surface recession of the TPS material. The shape change caused by surface recession can then impact the flow field, leading to a problem that involves strongly coupled fluid/solid interactions. Even in cases where there is no recession, the wall temperature profile resulting from aerodynamic heating can impact the state of the flow field. Therefore, when trying to predict the performance of an ablative TPS material, it is desirable to couple a flow model with a material response model in order to accurately capture the coupled nature of the problem.

In this study, the development of an axisymmetric material response model for non-charring materials is described, including verification studies. This material response model is then loosely coupled to an existing computational fluid dynamics (CFD) code through boundary conditions. To test the coupling procedure, simulations of the IRV-2 vehicle at several trajectory points are performed. The coupled results using the axisymmetric material response model are then compared with results from a one-dimensional material response model that is coupled in a similar manner to the same CFD code, and differences in both surface properties and in-depth heat transfer are observed. Finally, the results from both the one-dimensional and axisymmetric models are compared with results from the ABRES Shape Change Code (ASCC).

\section{Material Response}

\section{II.A. Overview}

The material response calculations are performed using a code that is being developed at the University of Michigan, and is based on MOPAR, ${ }^{1}$ a one-dimensional material response code also developed at the University of Michigan. The material response model uses the Control Volume Finite-Element Method $(\mathrm{CVFEM})^{2,3}$ to solve the energy equation shown in Equation 1, which includes a convection term due to grid motion to allow for mesh recession when ablation is occurring. Since the model is currently designed to be used solely for surface ablators, Equation 1 is the only conservation equation that is solved.

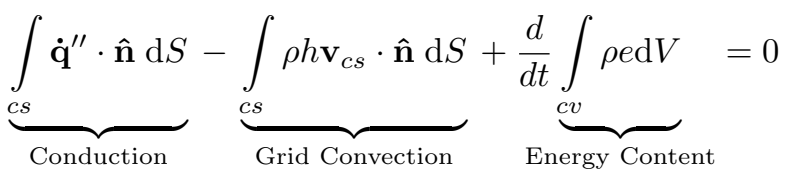

The heat flux is modeled using a tensor formulation of Fourier's Law to allow for simulation of anisotropic materials. The control surface velocity is determined from the change in node positions as the mesh is deformed. Newton's Method is used along with a preconditioned GMRES ${ }^{4}$ linear solver to solve the nonlinear energy equation, and the Backward Euler method is used for time integration. Linear triangular elements are used for the CVFEM, which leads to an implicit scheme that is second order accurate spatially and first order accurate temporally.

Several different boundary conditions are implemented including specified temperature, specified heat flux, re-radiation, aerodynamic heating, and thermochemical ablation. If a surface is ablating, the last three boundary conditions along with Equation 1 give the energy balance at the surface shown in Equation 2.

$$
\underbrace{-\overline{\mathbf{k}} \nabla T \cdot \hat{\mathbf{n}}}_{\text {Conduction }}-\underbrace{\rho_{w} h_{w} \mathbf{v}_{w} \cdot \hat{\mathbf{n}}}_{\text {Grid Convection }}=\underbrace{C_{h}\left(h_{r}-h_{w}\right)}_{\text {Convection }}-\underbrace{\epsilon \sigma\left(T_{w}^{4}-T_{\infty}^{4}\right)}_{\text {Reradiation }}-\underbrace{\rho_{s} \dot{s} h_{w} \hat{\mathbf{n}}}_{\text {Ablation }}
$$

The wall enthalpy and a dimensionless ablation mass transfer rate, $B_{c}^{\prime}$, are obtained from interpolating a thermochemical table which lists these quantities as functions of pressure and temperature. The surface recession rate can then be found from $B_{c}^{\prime}$ using Equation 3, where the mass transfer coefficient, $C_{m}$, is taken to be equal to $C_{h}$.

$$
\dot{s}=\frac{C_{m} B_{c}^{\prime}}{\rho_{s}}
$$

To move the solution forward a time step when there is ablation, the method of Hogan, Blackwell, and Cochran $^{5}$ is used to iteratively calculate $\dot{s}$, compute new temperatures, and move the mesh if necessary. This method iterates the thermal and mesh motion solutions at each time step until the change in surface temperature during that time step falls below some desired level. 


\section{II.B. Mesh Deformation}

In cases where there is surface recession, it is necessary to deform the mesh so that the numerical domain is coincident with the physical problem. In order to move an unstructured mesh in a consistent and general manner, the mesh is treated as a linear elastic solid, and the elastic solid mechanics equations are solved with zero body forces. ${ }^{5-7}$ For a two-dimensional domain, this leads to the equations shown below, which are written in terms of the nodal displacements $u, v$.

$$
\begin{aligned}
& \frac{1}{1-2 \nu} \frac{\partial}{\partial x}\left[(1-\nu) \frac{\partial u}{\partial x}+\nu\left(\frac{\partial v}{\partial y}\right)\right]+\frac{1}{2} \frac{\partial}{\partial y}\left(\frac{\partial u}{\partial y}+\frac{\partial v}{\partial x}\right)=0 \\
& \frac{1}{2} \frac{\partial}{\partial x}\left(\frac{\partial u}{\partial y}+\frac{\partial v}{\partial x}\right)+\frac{1}{1-2 \nu} \frac{\partial}{\partial y}\left[(1-\nu) \frac{\partial v}{\partial y}+\nu\left(\frac{\partial u}{\partial x}\right)\right]=0
\end{aligned}
$$

As can be seen, Poisson's ratio, $\nu$, is the only material property that is needed to solve these equations. Since the solution of Equation 4 is only used to move the mesh and does not affect the thermal solution (other than by possibly affecting the mesh quality), Poisson's ratio can be arbitrarily chosen. Following the work of Lynch and O'Neill, ${ }^{6} \nu$ is taken to be 0.0 for this study.

The solid mechanics equations are solved using the GMRES method with specified nodal displacements at the boundaries. At ablating boundaries, the nodal displacement over a time step is found using the surface recession rate calculated in Equation 3, and is given by

$$
\left[\begin{array}{l}
u \\
v
\end{array}\right]=\left[\begin{array}{l}
\dot{s} \Delta t n_{x} \\
\dot{s} \Delta t n_{y}
\end{array}\right]
$$

where $n_{x}, n_{y}$ are the components of the surface unit normal vector, $\hat{\mathbf{n}}$. All other non-ablating boundaries are assumed to have zero displacement. Non-ablating boundary nodes near an ablating surface, however, are allowed to slide along the boundary. This prevents elements near the ablating surface from becoming highly skewed.

\section{II.C. Verification}

To ensure that the solver is correctly implemented and that Equation 1 and its boundary conditions are correctly discretized, the verification studies found in $\mathrm{Amar}^{2}$ and Amar, Calvert, and Kirk ${ }^{8}$ for transient conduction with variable properties and for the radiative boundary condition are used. Note that at this time neither the grid convection term of the energy equation nor the general thermochemical boundary condition have been formally verified.

The transient conduction test consists of a square domain with a constant specified heat flux on one edge, and with an adiabatic condition on the other edges. The normalized $L_{2}$ norm of the nodal temperature error, defined below, is used as the error metric with $T_{o}=300 \mathrm{~K}$.

$$
\text { error }=\frac{1}{T_{o}} \sqrt{\frac{1}{N} \sum_{n=1}^{N}\left(T_{\text {exact }}-T_{\text {calc }}\right)^{2}}
$$

Details of the grids used in the verification study are listed in Table 1. Note that all meshes are made up of triangular elements, and each successive mesh is a uniform refinement of the previous mesh. Also note that the time steps are chosen so that the spatial and temporal orders of accuracy can be simultaneously verified. ${ }^{8}$ Results are presented as functions of the number of elements in one dimension, which are found for a square domain as

$$
\text { Elements in One Dimension }=\sqrt{N_{e}}
$$

where $N_{e}$ is the total number of elements. In addition to verifying the spatial and temporal orders of accuracy, the implementation of Newton's method is also verified for both the transient conduction and radiative boundary condition cases. Verifying Newton's method serves to check that the terms of the Jacobian matrix are correct within the code. The error metric in this case is the relative $L_{2}$ norm of the independent variable correction vector. ${ }^{8}$ 


\begin{tabular}{ccccc}
\hline \hline Grid & Nodes & Total Elements & "1D" Elements & $\Delta t$, seconds \\
\hline Initial & 20 & 26 & 5.1 & 1.0 \\
Coarse & 65 & 104 & 10.2 & 0.5 \\
Medium & 233 & 416 & 20.4 & 0.25 \\
Fine & 881 & 1664 & 40.8 & 0.125 \\
\hline \hline
\end{tabular}

Table 1: Mesh parameters for verification studies.

Results for the order of accuracy and Newton's method verification of the conduction term at a simulated time of $t=1.0 \mathrm{~s}$ are shown below in Figure 1. In both cases, the expected second-order rate of convergence is obtained, thereby verifying the implementation of this term.

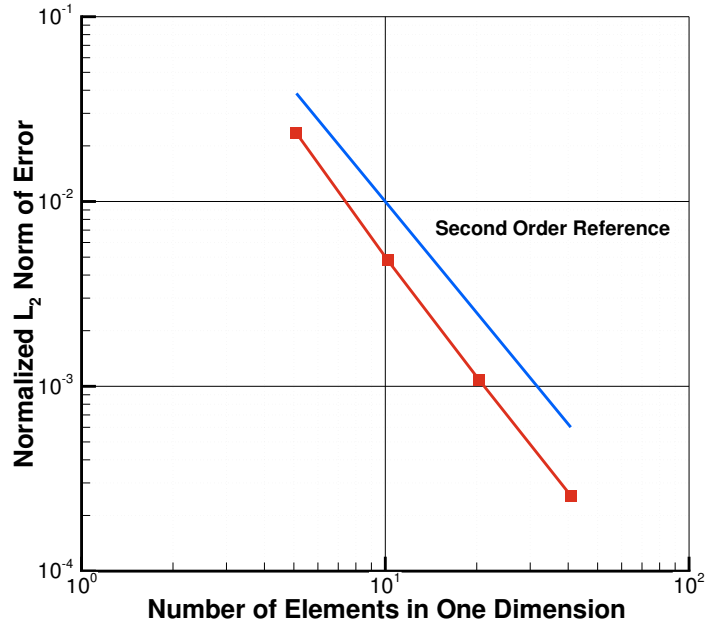

(a) Grid Refinement

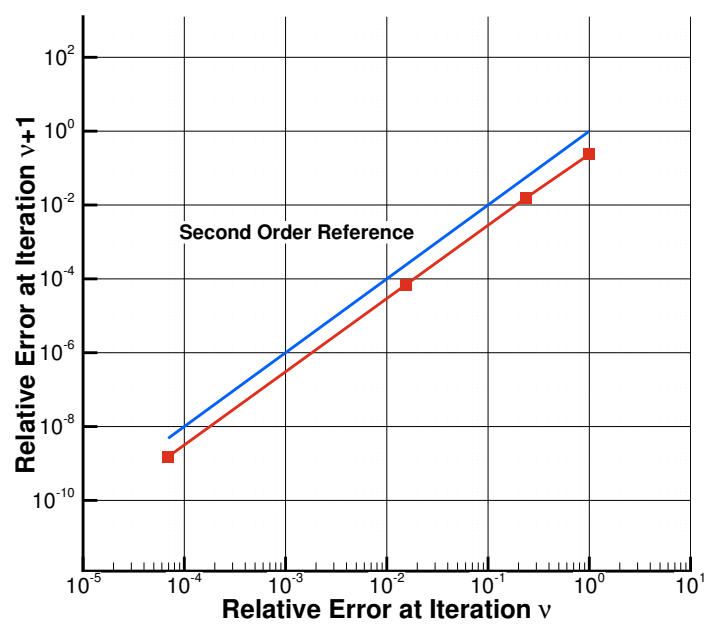

(b) Newton's Method

Figure 1: Verification of the transient conduction term at $t=1.0$ seconds.

The radiative boundary condition test is similar to the transient conduction case, except that the applied flux is given by the reradiation term in Eq. 2, and a steady state solution is sought, rather than a transient solution. For the verification case presented here, $T_{\infty}=1300 \mathrm{~K}$ and $T_{w}=300 \mathrm{~K}$. The error metric for this case is the temperature on the front face of the domain, where the radiative flux is applied. From Amar, ${ }^{2}$ this temperature should be constant across the face, and a second-order discretization should reproduce this temperature to within machine precision without any mesh refinement.

The results of the Newton's method convergence rate study for the radiative boundary condition are shown in Figure 2. As can be seen, the convergence rate is second-order as expected. Also, although it's not shown here, the front face temperature is correctly predicted to within machine precision without mesh refinement, indicating that the boundary condition is correctly implemented. 


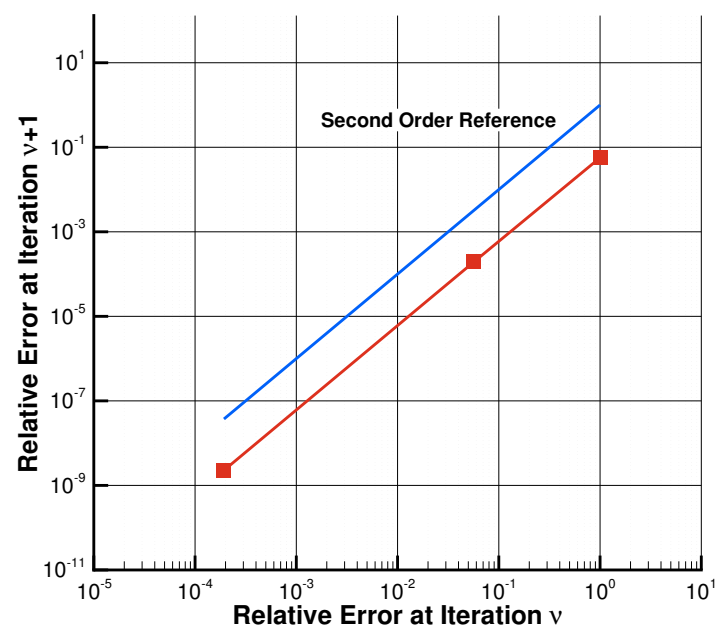

Figure 2: Verification of Newton's method convergence for the radiative boundary condition.

\section{Hypersonic Flow Code}

The CFD code used in this study is LeMANS, ${ }^{9,10}$ which is a laminar Navier-Stokes solver developed at the University of Michigan for use on hypersonic problems. It is capable of simulating flows with both thermal (translation/rotational/vibrational) and chemical non-quilibrium. The translational and rotational energy modes of all species are described by the temperatures $T$ and $T_{\text {rot }}$ respectively, and the combined vibrational and electronic energies of all species are described by a single temperature, $T_{v e} \cdot{ }^{11}$ Viscous stresses are modeled assuming a Newtonian fluid and using Stokes' hypothesis. Transport properties are modeled either with Wilke's semi-empirical mixing rule using Blottner's curve fits for viscosity and Eucken's relation for thermal conductivities, or with Gupta's mixing rule using non-coulombic/coulombic collision cross section data to calculate viscosities and thermal conductivities. For this study the former method is used. The heat flux to a vehicle's surface is made up of a convective term, modeled using Fourier's law for all temperatures, and a mass diffusion term modeled using a modified form of Fick's Law. ${ }^{12}$ The surface can be treated as either non-catalytic or super-catalytic, and, in addition, ablative species can be introduced via a blowing boundary condition. ${ }^{13}$

LeMANS is capable of simulating two-dimensional/axisymmetric flows using any mix of triangles and quadrilaterals in the mesh, and three-dimensional flows using hexahedra, tetrahedra, prisms, and pyramids. Time integration is performed using either a point or line implicit method, and numerical fluxes are modeled using a modified Steger-Warming Flux Vector Splitting scheme which has low dissipation in boundary layer regions. LeMANS is capable of running on parallel machines and uses METIS ${ }^{14}$ to partition the mesh and MPI for inter-processor communication.

Mesh movement has also been implemented in LeMANS to handle material response cases that include surface recession. The algorithm for moving the mesh is described in Martin and Boyd. ${ }^{15}$ The basic idea is to identify lines in the mesh that are normal to the vehicle's surface, non-dimensionalize the node positions along the lines using the total line length (similar to Landau coordinates), and then linearly adjust each node's position based on the amount of surface recession. This method works well for the simple, smooth geometries often considered when studying hypersonic flight, but it is not general enough to handle arbitrary three-dimensional geometries.

\section{Coupling of Material Response and External Flow}

The material response model is implemented as a boundary subroutine of LeMANS in order to link the flow and surface processes. This method of coupling CFD and material response has been previously demonstrated for LeMANS and the one-dimensional MOPAR code. ${ }^{13,15,16}$ In this method a vehicle's trajectory is discretized into several points. A steady state solution is then found at each trajectory point using the 
solution from the previous trajectory point as the initial condition. As the CFD solution of the flow field is marched to steady state, the material response subroutine is called after a set number of flow field iterations. For example, in this study the material response is called every 100 flow field iterations. When the material response is called, the aerodynamic heating boundary condition shown in Equation 8 is used to compute the convective heat flux applied to a surface.

$$
q_{a h}=\rho C_{h}\left(h_{w}-h_{r}\right)
$$

The heat flux from LeMANS is used to compute $C_{h}$ using Equation $9 .^{17}$

$$
C_{h}=\frac{q_{C F D}}{h_{r}-h_{w}}
$$

As previously mentioned, the wall enthalpy is determined as a function of pressure (from LeMANS) and temperature by interpolating a thermochemical table, and the recovery enthalpy is assumed to be equal to the freestream stagnation enthalpy. Once the boundary condition is specified, the material response is then modeled in a time-accurate fashion from trajectory point $i$ to $i+1$ with the applied heat flux and wall pressure being linearly interpolated from the intial condition to the current value calculated by LeMANS. Once the material response has been modeled up to trajectory point $i+1$, the updated wall temperature, mass flux, and wall position are then passed back to LeMANS, and the flowfield is iterated for a set number of iterations before the material response is called again. A diagram of the coupling procedure is shown in Figure 3.

In order to provide flexibility when generating meshes, the nodes of the fluid and solid meshes at the mesh interface are not required to be coincident. So while the surface shape will be the same in both meshes, the number elements can be different. This allows for independent refinements of the flow and solid meshes tailored to the problem that is being solved. One downside of this approach, however, is that it is necessary to interpolate values between the two meshes. To accomplish this, a version of Shepard's method ${ }^{18,19}$ is used. This is an inverse-distance weighting method that uses data within some radius, $R$, of the location where an interpolated value is desired. The initial search radius is increased until a user-defined

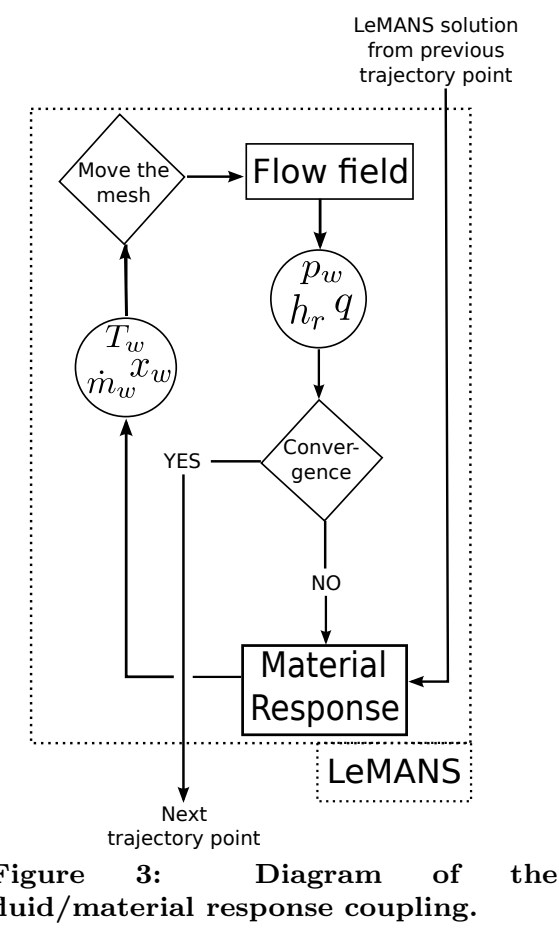
minimum number of interpolating points are found. This method is very general and can easily be extended to three-dimensional geometries.

\section{Results}

To demonstrate the coupling method, the axisymmetric, carbon-carbon nosetip of the IRV-2 vehicle ${ }^{20}$ is considered. This test case allows for comparison with one-dimensional material response data obtained from coupling LeMANS with MOPAR. This case also allows for comparison with the ASCC code, ${ }^{21}$ which incorporates flight data and is therefore considered a useful benchmark.

The initial meshes used are shown in Figure 4. At the boundary between the two meshes, a wall boundary condition is applied in the flow model with the wall conditions obtained from the material response solution. In the material response model, aerodynamic heating, re-radiation, and ablation boundary conditions are applied. At the back-face of the solid, an adiabatic boundary is assumed since the actual back wall temperatures are unknown.

In the following results, the IRV-2 reentry trajectory is split into several discrete points, ${ }^{17}$ and solutions are obtained at each point. The freestream conditions for the first four trajectory points are shown in Table 2. At each of these points, a steady state coupled solution is sought that takes into account the solutions at previous trajectory points, and this is performed via the following procedure. First, trajectory point 0 is run without material response and assuming an isothermal wall at the freestream temperature. Once a 


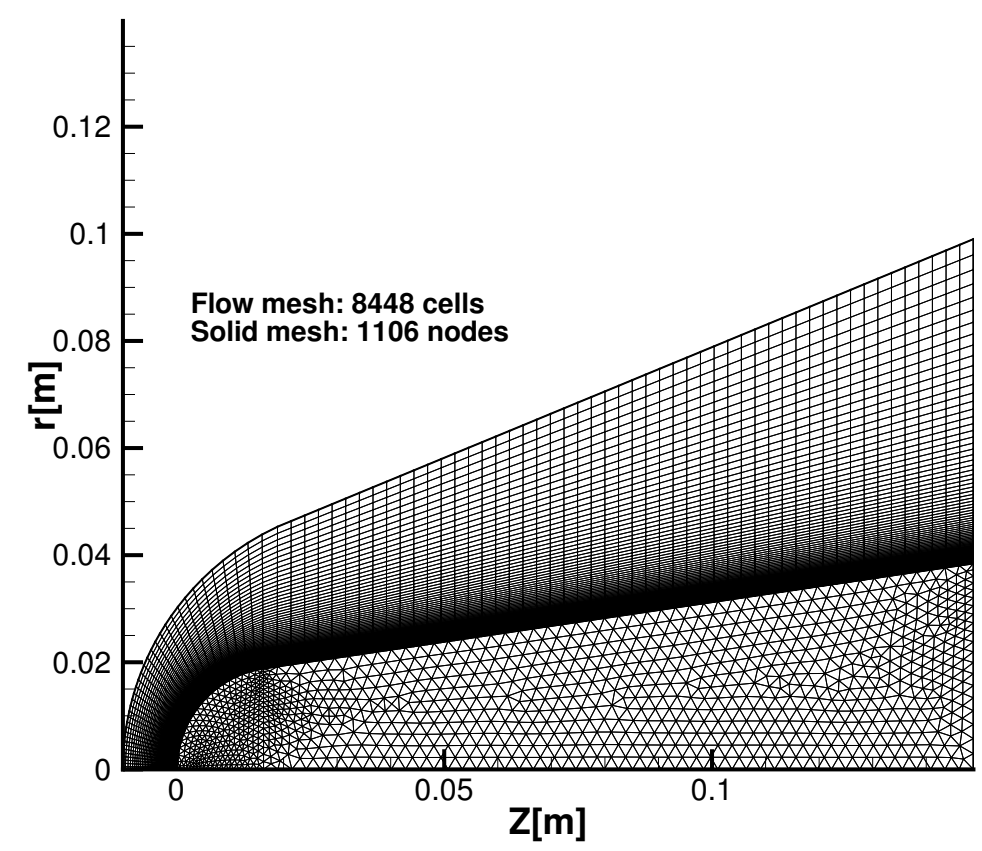

Figure 4: Initial flow and solid meshes for the IRV-2 simulations.

converged flow field solution at point 0 is obtained, the flow field of trajectory point 1 is then initialized with the solution from point 0 , and the solid at point 1 is initialized to a temperature of $300 \mathrm{~K}$. A coupled steady state solution is then found for point 1 . For the next trajectory points, both the flow and material response models are initialized to the solution from the previous trajectory point and the freestream values are used as boundary conditions. In this way, the entire reentry trajectory can be simulated one point at a time.

\begin{tabular}{cccccc}
\hline \hline Trajectory point & $\begin{array}{c}\text { Reentry time } \\
{[\mathrm{s}]}\end{array}$ & $\begin{array}{c}\text { Altitude } \\
{[\mathrm{km}]}\end{array}$ & Mach No. & $\begin{array}{c}\text { Temperature } \\
{[\mathrm{K}]}\end{array}$ & $\begin{array}{c}\text { Density } \\
{\left[\mathrm{kg} / \mathrm{m}^{3}\right]}\end{array}$ \\
\hline 0 & 0.00 & 66.7 & 22 & 227.8 & $1.25 \times 10^{-4}$ \\
1 & 4.25 & 56.0 & 21 & 258.0 & $5.05 \times 10^{-4}$ \\
2 & 6.75 & 49.3 & 21 & 270.7 & $1.13 \times 10^{-3}$ \\
3 & 8.75 & 44.0 & 21 & 261.4 & $2.26 \times 10^{-3}$ \\
\hline \hline
\end{tabular}

Table 2: Freestream conditions for the IRV-2 reentry trajectory.

Using this approach, the trajectory points shown in Table 2 are simulated. Figure 5 shows the temperature contours for the axisymmetric material response at trajectory points 1 and 3 . As can be seen from the figure, the surface recession remains smooth, and the flow mesh remains attached to the solid throughout the ablation process. This is important since it indicates that the mechanics of the mesh motion procedure are working as intended. One potential problem, however, is that as the flow mesh is moved, accurate alignment of the mesh with the shock is not guaranteed. This can negatively impact the prediction of surface quantities and a means of maintaining shock alignment and resolution on a moving mesh needs to be studied further.

Figure 6 shows another comparison of temperature contours, but this time at $t=4.25 \mathrm{~s}$ for the one dimensional and axisymmetric material response results. The one dimensional results are obtained at each boundary cell in the flow mesh along a line that is normal to the surface and has a constant length. The 


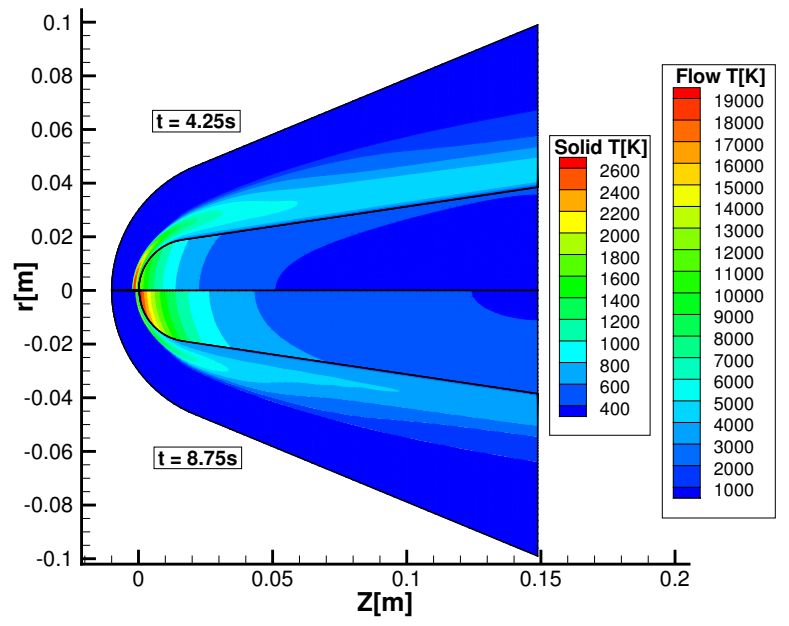

(a) Full view

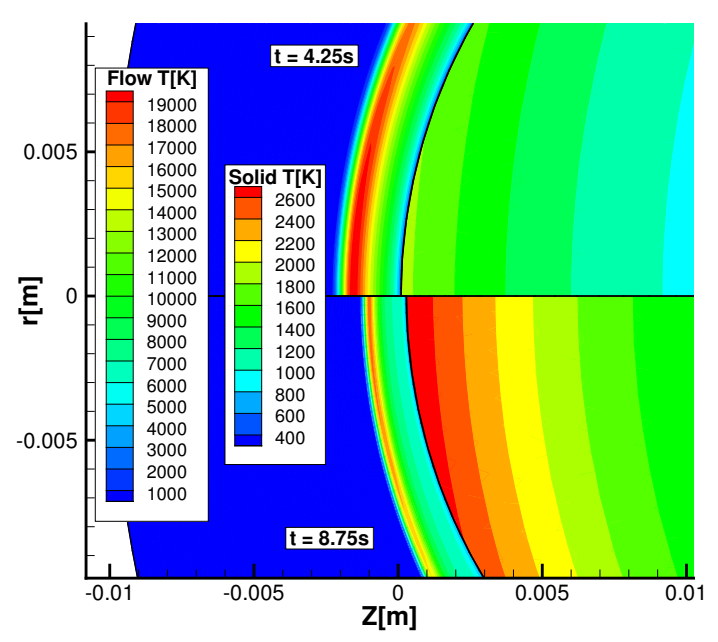

(b) Close-up of stagnation region

Figure 5: Temperature contours for the axisymmetric solution.

contours are then generated by interpolating all of the one dimensional solutions together.

The flow field results are nearly identical to one another, which is not surprising since in both cases the flow solution is axisymmetric and the surface values are not drastically different. The solid solutions are noticeably different, however, especially in the shape of the temperature contours.

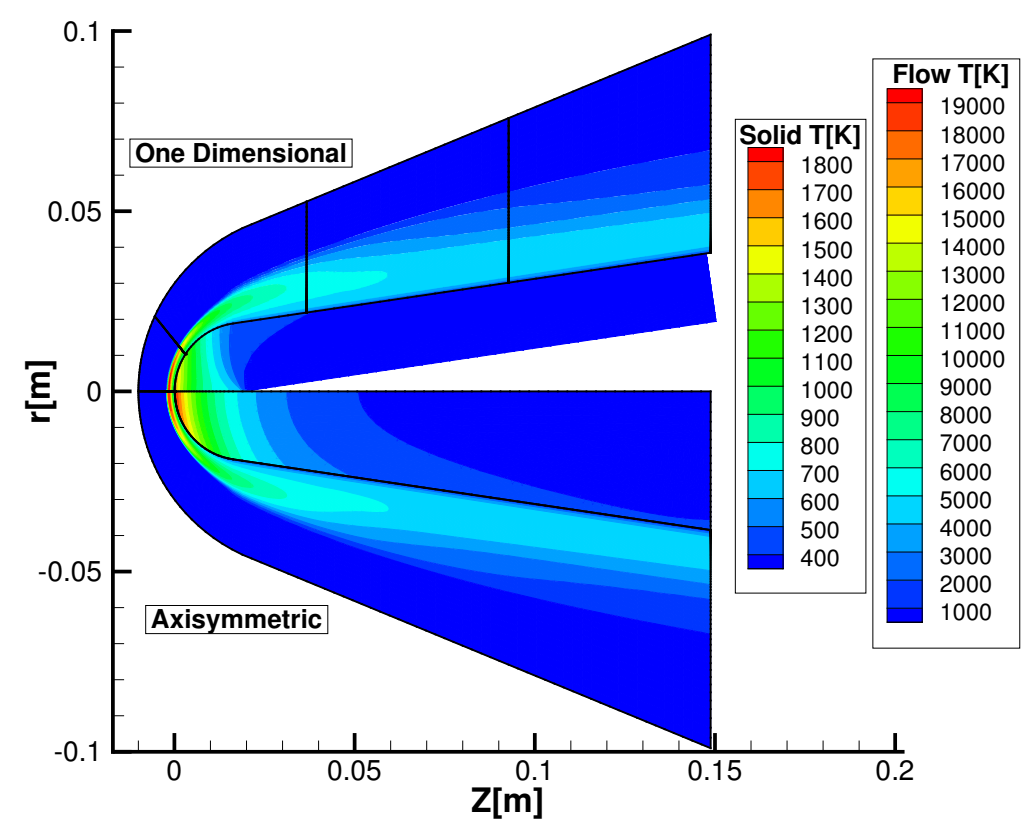

Figure 6: Comparison of $1 \mathrm{D}$ and axisymmetric results at $t=4.25 \mathrm{~s}$.

To further examine the differences between the one dimensional and axisymmetric material response models, the temperature contours in the solid are shown for the last three trajectory points in Figure 7 . The two-dimensional nature of the heat transport is clearly evident from the temperature contours, and this leads to a couple of observations. First, near the vehicle's surface, the axisymmetric contours are more curved. 
This causes higher temperatures to be found further aft along the surface than for the one-dimensional model. Second, along the stagnation line $(r=0 m)$ the one-dimensional contours become elongated with high temperatures penetrating further into the material than they should. This is a result of the high temperatures near the stagnation point being unable to diffuse into the material. In the axisymmetric case this problem is alleviated, leading to a more physically accurate prediction of the in-depth material response.

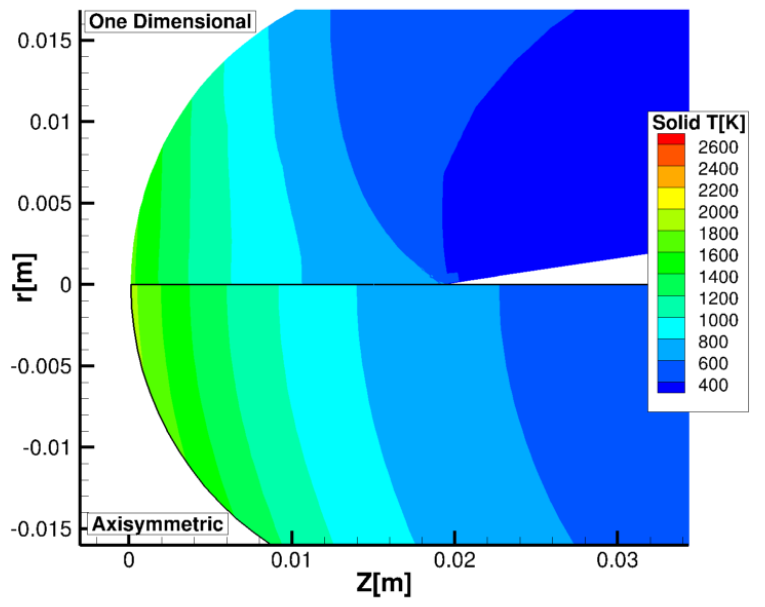

(a) $t=4.25 \mathrm{~s}$

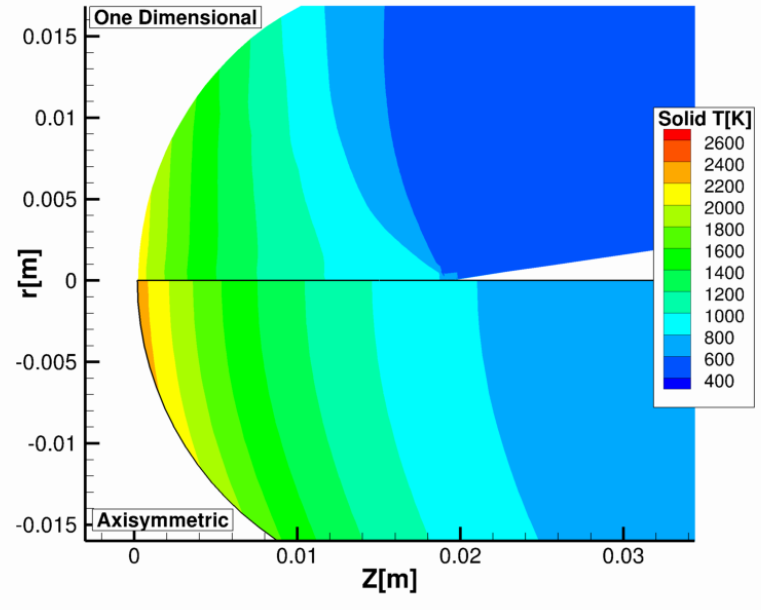

(b) $t=6.75 \mathrm{~s}$

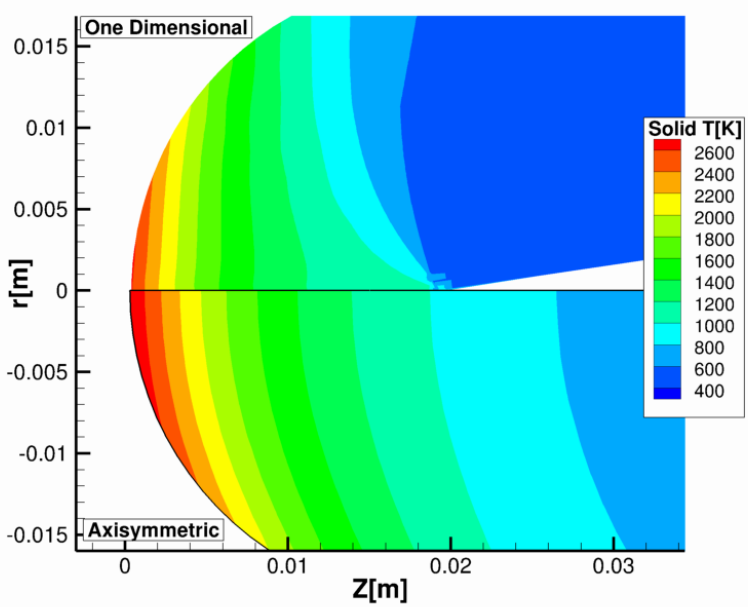

(c) $t=8.75 \mathrm{~s}$

Figure 7: Comparison of one-dimensional and axisymmetric temperature contours.

Looking more closely at vehicle surface properties, Figure 8 shows the comparison between one-dimensional and axisymmetric results for several different quantities. The heat flux, ablating mass flux, and rate of recession predicted by the one-dimensional approach are all higher at the stagnation point than the corresponding axisymmetric results. However, the stagnation point temperature of the one-dimensional method is lower than that obtained with the new axisymmetric approach. This circumstance is explained by the significantly higher level of thermal conductivity within the material that is predicted using the one-dimensional description and that arises from stronger temperature gradients.

Similar to the temperature data, the blowing rates also show a broader profile in the axisymmetric results than in the one-dimensional results. Unlike temperature, however, near the stagnation point the one-dimensional model has a higher blowing rate than the axisymmetric model. The blowing rate differences 
lead to different surface shapes with the one-dimensional model predicting more surface recession near the stagnation point, but less as the radial coordinate increases. This is a result of the broader blowing rate profiles in the axisymmetric material response which indicate that ablation is occurring further aft along the IRV-2 surface than in the one-dimensional case. So, while the axisymmetric case predicts less surface recession near the stagnation point than the one-dimensional case, it predicts some surface recession over a greater percentage of the vehicle.

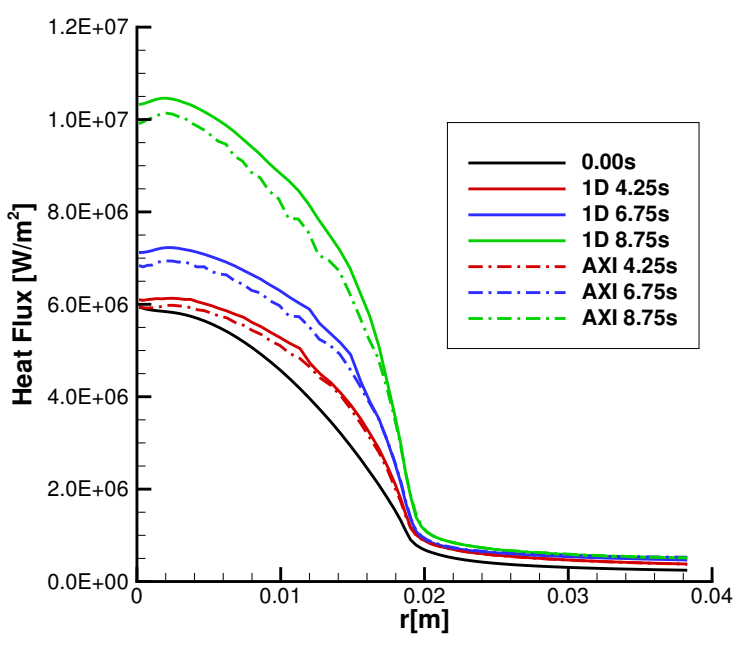

(a) Heat flux

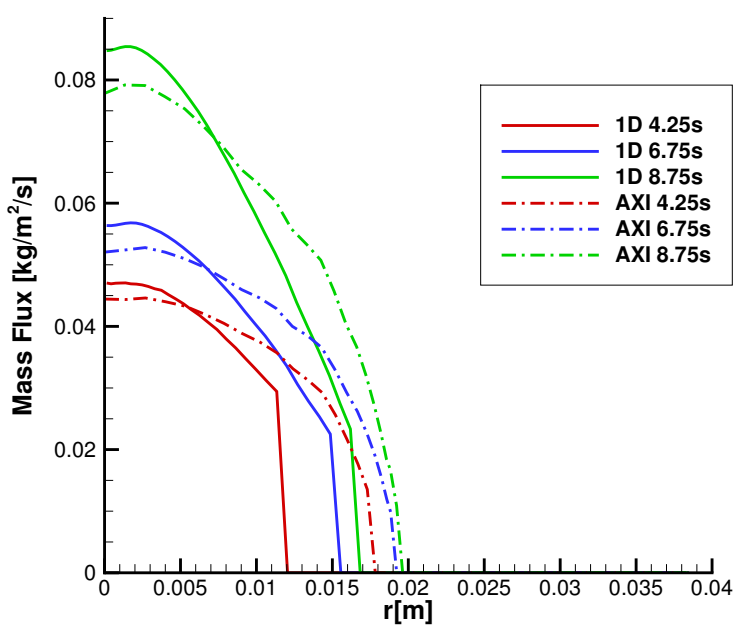

(c) Blowing rates

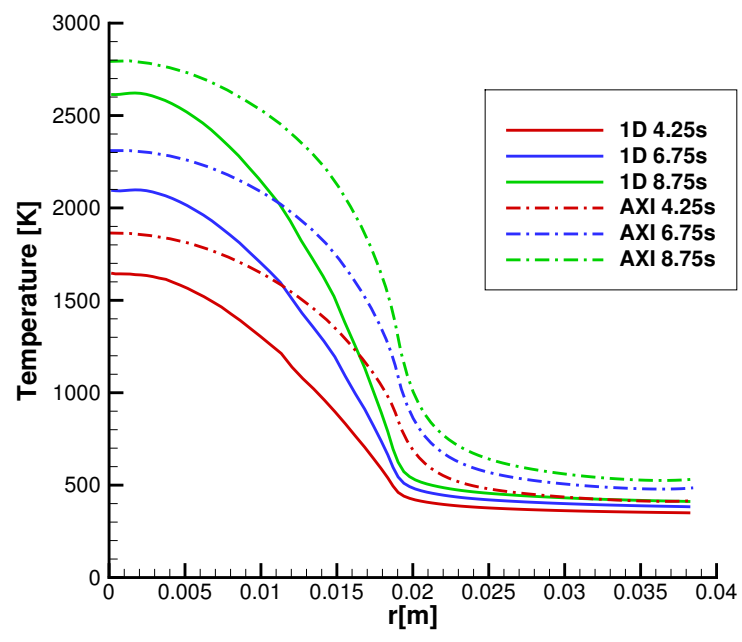

(b) Temperature

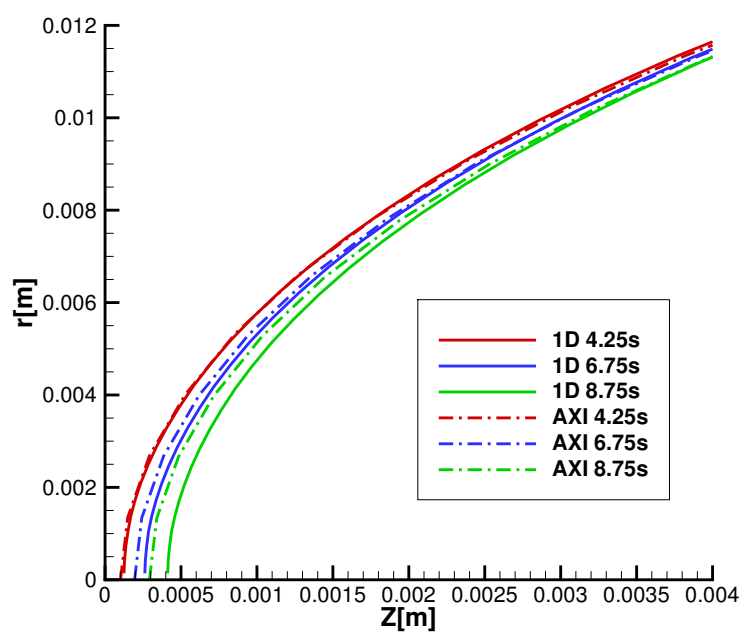

(d) Surface shape

Figure 8: Comparison of several different surface properties between one-dimensional and axisymmetric results.

In addition to comparing results between the one-dimensional and axisymmetric material response codes, the axisymmetric code is also compared against results ${ }^{17}$ obtained with the ASCC code. Figure 9a compares the predicted stagnation point temperatures between ASCC and both the axisymmetric and the one-dimensional LeMANS/material response coupled results. Both models predict lower stagnation point temperatures than ASCC for all trajectory points, and the agreement with ASCC becomes worse for both codes at each successive trajectory point. Previous results ${ }^{15}$ for the one-dimensional model show higher temperatures than ASCC, which is due to using a super-catalytic wall boundary condition, as opposed to 
the non-catalytic boundary used in the current study.

Figure 9b shows a comparison of the stagnation point surface recession between the axisymmetric model and ASCC. ASCC predicts a higher surface recession rate with an increasing difference between LeMANS and ASCC for later trajectory points. The differences in both of these results may arise from many different sources, but two likely causes are quality issues for the flow mesh at later trajectory points, and the fact that the exact material properties used in the ASCC code are not known to the authors. For the results obtained in the present study, a generic carbon-carbon material was assumed, which may have different properties than that used in ASCC. The mesh quality issues arise from the fact that as the vehicle surface recesses and the flow mesh is moved, the shock is no longer resolved as well as it was on the initial mesh. In particular, the mesh is no longer aligned as accurately with the shock.

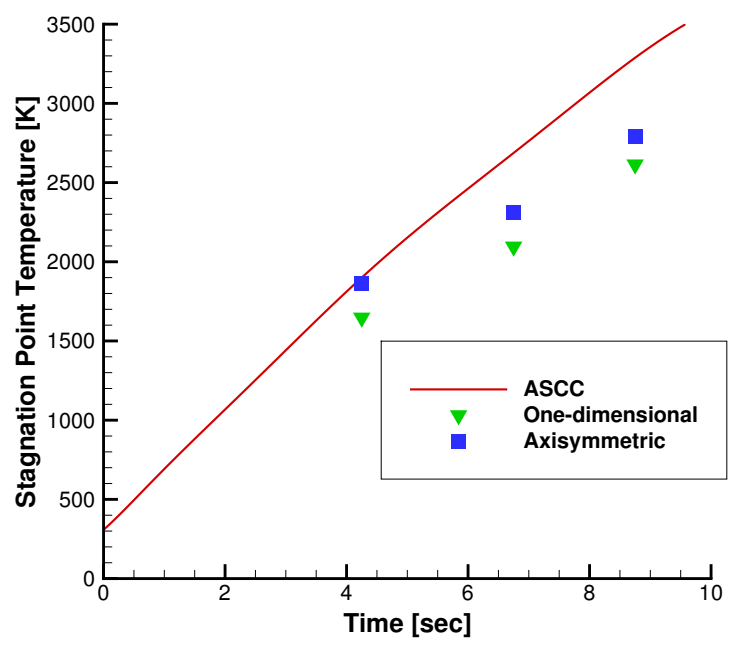

(a) Stagnation point temperatures

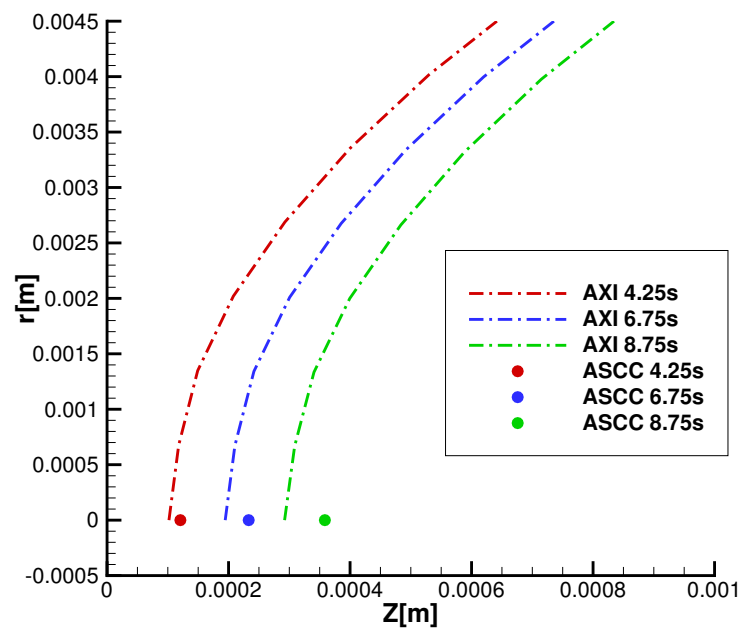

(b) Surface recession

Figure 9: Comparison of stagnation point quantities between ASCC and the LeMANS/material response coupled results.

\section{Conclusions and Future Work}

The capability to accurately model and predict the complex physical phenomena that occur during hypersonic flight is important to improve the design of these vehicles. To improve modeling capabilities, a two-dimensional/axisymmetric material response model was presented, and subsequently coupled to a hypersonic CFD code. This allowed for fully coupled fluid/solid simulations of the aerothermal environment of an ablating hypersonic vehicle. The material response code and the coupling process were tested on the IRV-2 vehicle for several points along its trajectory, and the results compared with a one-dimensional material response code. In general, results from the one-dimensional and axisymmetric models were similar, however, the effects of multi-dimensional heat transport were very evident near the vehicle nose. In addition, the axisymmetric results were compared with ASCC and it was found that ASCC predicted higher stagnation point temperatures and recession rates.

To further improve this method, some way of maintaining the quality of the flow field mesh as a vehicle surface recesses is necessary. In particular, a method for keeping the mesh aligned with the shock needs to be implemented since poor alignment can lead to inaccurate surface flux calculations. In addition, it is desirable to extend both the material response calculations and the coupling process to fully three-dimensional geometries. Also, adding the ability to compute the stress and strain fields introduced to the vehicle during flight will provide a fuller picture of material behavior at high temperatures, and will further help to improve the design process of hypersonic vehicles. 


\section{Acknowledgments}

The authors would like to thank Alexandre Martin for his help in obtaining the MOPAR results, and also for several helpful discussions. Funding is provided to the first author by the Air Force Research Laboratory (AFRL) through the Collaborative Center in Aeronautical Sciences (CCAS).

\section{References}

${ }^{1}$ Martin, A. and Boyd, I. D., "Simulation of pyrolysis gas within a thermal protection system," 40th AIAA Thermophysics
Conference, Seattle, Washington, June 23-26 2008, AIAA paper 2008-3805.
${ }^{2}$ Amar, A. J., Modeling of One-Dimensional Ablation with Porous Flow Using Finite Control Volume Procedure, Master's
thesis, North Carolina State University, 2006.
${ }^{3}$ Blackwell, B. and Hogan, R., "Numerical Solution of Axisymmetric Heat Conduction Problems Using Finite Control Volume Technique," Journal of Thermophysics and Heat Transfer, Vol. 7, No. 3, 1993, pp. 462-471.

${ }^{4}$ Saad, Y. and Schultz, M. H., "GMRES: A Generalized Minimal Residual Algorithm for Solving Nonsymmetric Linear Systems," SIAM Journal on Scientific and Statistical Computing, Vol. 7, No. 3, July 1986, pp. 856-869.

${ }^{5}$ Hogan, R., Blackwell, B., and Cochran, R., "Application of Moving Grid Control Volume Finite Element Method to Ablation Problems," Journal of Thermophysics and Heat Transfer, Vol. 10, No. 2, April-June 1996, pp. 312-319.

${ }^{6}$ Lynch, D. and O'Neill, K., "Elastic Grid Deformation for Moving Boundary Problems in Two Space Dimensions," Finite Elements in Water Resources, Vol. 2, 1980, pp. 7.111-7.120.

${ }^{7}$ Dec, J. A., Three Dimensional Finite Element Ablative Thermal Response Analysis Applied to Heatshield Penetration Design, Ph.D. thesis, Georgia Institute of Technology, 2010.

${ }^{8}$ Amar, A., Calvert, N., and Kirk, B., "Development and Verification of the Charring Ablating Thermal Protection Implicit System Solver," 49th AIAA Aerospace Sciences Meeting including the New Horizons Forum and Aerospace Exposition, Orlando, Florida, January 4-7 2011, AIAA paper 2011-144.

${ }^{9}$ Scalabrin, L. D., Numerical Simulation of Weakly Ionized Hypersonic Flow Over Reentry Capsules, Ph.D. thesis, University of Michigan, 2007.

${ }^{10}$ Martin, A., Scalabrin, L. C., and Boyd, I. D., "High Performance Modeling of Atmospheric Re-entry Vehicles," Journal of Physics: Conference Series, Vol. 341, 2012, Article 012002.

${ }^{11}$ Holman, T. D. and Boyd, I. D., "Effects of Continuum Breakdown on the Surface Properties of a Hypersonic Sphere," Journal of Thermophysics and Heat Transfer, Vol. 23, 2009, pp. 660-673.

${ }^{12}$ Sutton, K. and Gnoffo, P., "Multi-Component Diffusion with Application to Computational Aerothermodynamics," AIAA/ASME Joint Thermophysics and Heat Transfer Conference, Albuquerque, NM, June 15-18 1998, AIAA paper 19982575.

${ }^{13}$ Martin, A. and Boyd, I. D., "Strongly coupled computation of material response and nonequilibrium flow for hypersonic ablation," 41st AIAA Thermophysics Conference, San Antonio, Texas, June 22-25 2009, AIAA paper 2009-3597.

${ }^{14}$ Karypis, G. and Kumar, V., METIS: A Software Package for Partitioning Unstructured Graphs, Partitioning Meshes, and Computing Fill-Reducing Orderings of Sparse Matrices, University of Minnesota, Minneapolis, MN, version 4.0 ed., September 1998 .

${ }^{15}$ Martin, A. and Boyd, I. D., "Implicit implementation of material response and moving meshes for hypersonic re-entry ablation," 47th AIAA Aerospace Sciences Meeting and Exhibit, Orlando, Florida, January 5-8 2009, AIAA paper $2009-0670$.

${ }^{16}$ Martin, A. and Boyd, I. D., "Mesh tailoring for strongly coupled computation of ablative material in nonequilibrium hypersonic flow," 10th AIAA/ASME Joint Thermophysics and Heat Transfer Conference, Chicago, Illinois, June 28 - July 1 2010, AIAA paper 2010-5062.

${ }^{17}$ Kuntz, D. W., Hassan, B., and Potter, D. L., "Predictions of Ablating Hypersonic Vehicles Using an Iterative Coupled Fluid/Thermal Approach," Journal of Thermophysics and Heat Transfer, Vol. 15, No. 2, 2001, pp. 129-139.

${ }^{18}$ Shepard, D., "A two-dimensional interpolation function for irregularly-spaced data," Proceedings of the 1968 23rd ACM national conference, ACM '68, ACM, New York, NY, USA, 1968, pp. 517-524.

${ }^{19}$ Franke, R., "Scattered Data Interpolation: Tests of Some Methods," Mathematics of Computation, Vol. 38, No. 157, January 1982.

${ }^{20}$ Hassan, B., Kuntz, D. W., Salguero, D. E., and Potter, D. L., "A Coupled Fluid/Thermal/Flight Dynamics Approach for Predicting Hypersonic Vehicle Performance," 35th Thermophysics Conference, Anaheim, California, June 11-14 2001, AIAA paper 2001-2903.

${ }^{21}$ King, H., Muramoto, K., Murray, A., and Pronchick, S., ABRES Shape Change Code (ASCC86) - Technical Report and User's Manual, Acurex Corp., Aerotherm Div., Mountain View, CA, December 1986, Rept. BMO TR-87-57. 